\title{
Au carrefour de l'écologie industrielle et du Syal. Premiers jalons pour faire progresser la durabilité d'un développement rural localisé
}

At the crossroads of industrial ecology and Syal First steps to advance the sustainability of a Localized rural development

\section{Renaud Metereau et Catherine Figuière}

\section{OpenEdition}

Journals

Édition électronique

URL : http://journals.openedition.org/developpementdurable/10123

DOI : 10.4000/developpementdurable.10123

ISSN : 1772-9971

Éditeur

Association DD\&T

Référence électronique

Renaud Metereau et Catherine Figuière, «Au carrefour de l'écologie industrielle et du Syal. Premiers jalons pour faire progresser la durabilité d'un développement rural localisé », Développement durable et territoires [En ligne], Vol. 5, nº | Février 2014, mis en ligne le 04 février 2014, consulté le 01 mai 2019. URL : http://journals.openedition.org/developpementdurable/10123; DOI : 10.4000/ developpementdurable.10123

Ce document a été généré automatiquement le 1 mai 2019.

Développement Durable et Territoires est mis à disposition selon les termes de la licence Creative Commons Attribution - Pas d'Utilisation Commerciale 4.0 International. 


\title{
Au carrefour de l'écologie industrielle et du Syal. Premiers jalons pour faire progresser la durabilité d'un développement rural localisé ${ }^{1}$
}

\author{
At the crossroads of industrial ecology and Syal First steps to advance the \\ sustainability of a Localized rural development
}

Renaud Metereau et Catherine Figuière

1 L'année 2008 a définitivement confirmé l'ouverture d'une période de réaffirmation de la question agricole, non seulement dans la réflexion des économistes, mais plus généralement dans celle de tous les acteurs du développement. Sur fond de crise alimentaire mondiale et suite à la parution du rapport de la Banque Mondiale (2008) « L'agriculture au service du développement ", l'agriculture et l'alimentation constituent à nouveau un socle incontournable pour l'élaboration de stratégies de lutte contre la pauvreté. Effectivement, le secteur agricole n'avait jusqu'à présent été que trop marginalement pris en compte en tant que déterminant des dynamiques de développement: "L'agriculture présente un paradoxe apparent: elle contribue pour une part importante de l'activité économique des pays pauvres, mais le développement agricole est rarement pensé comme un moteur de la croissance économique [et avant tout de réduction de la pauvreté]. L'accent mis sur le décollage industriel a pu amener à négliger les politiques agricoles, à sous-estimer l'interaction entre les gains de productivité dans le secteur rural, la croissance des villes et l'émergence des activités industrielles et de service, à concentrer l'aide publique au développement sur d'autres secteurs que l'agriculture. » (Vindel et Jacquet, 2011 : 73).

2 L'objectif de cette contribution est clairement programmatique: il s'agit de poser les premiers jalons d'une réflexion visant à faire progresser la durabilité d'un «développement rural local» (DRL), en combinant les «démarches de type Syal» 
(Système Agroalimentaire Localisé) avec le principe de bouclage des flux (de matières et d'énergie) de l'écologie industrielle (EI).

3 La démarche mobilisée s'inscrit dans une conception du développement durable anthropocentrée (Sachs, 1980) et en durabilité forte, tout en accordant une place centrale au territoire comme espace de mise en œuvre (Theys, 2002). Elle est donc également en accord avec la proposition d'une "socio ecological economics" portée par Spash (2011).

4 Afin de poser les bases du « développement rural local durable »DRLD, le Syal est ici choisi comme "brique de base $»^{2}$ (partie 1), bien que cette notion n'ait qu'une capacité postulée, et non démontrée, à intégrer la dimension environnementale (partie 2). Dans le but de rendre plus durable le projet de développement local dont il est initialement porteur, nous allons tout d'abord "compléter" le Syal avec des outils spécifiques aux activités agricoles et à visée explicitement environnementale : l'agroécologie et l'IFES ( Integrated Food Energy System) (partie 3). Nous montrerons ensuite en quoi les principes fondateurs de l'écologie industrielle permettent de systématiser le principe de bouclage de flux de matières et d'énergie, déjà suggéré dans le projet d'IFES (partie 4). La partie 5 s'arrêtera sur les spécificités de la lecture socio-économique de l'EI, qui va s'avérer particulièrement compatible avec les propositions du Syal, lorsque ce dernier est conçu comme un «levier stratégique » pour le développement local (partie 6).

\section{Le Système Agroalimentaire Localisé, « brique de base » du DRLD.}

5 Notre proposition prend le Syal comme « brique de base », qu'il s'agit de faire progresser pour en faire un outil de DRLD. La notion de Syal apparait au milieu des années 1990 pour caractériser certaines formes d'organisations localisées et de coopérations en milieu rural entre petites entreprises et petites agro-industries rurales. Cette notion émerge donc du rapprochement entre des travaux empiriques réalisés notamment en Amérique Latine et en Afrique Subsaharienne d'une part, et des approches localisées du développement économique, marshalliennes ou "néo-marshalliennes ", ayant abouti à la formation du concept de Système Productif Localisé (Courlet, 2008) d'autre part. Il s'agit alors de doter d'un cadre analytique certaines formes de regroupements localisés de petites entreprises de l'agroalimentaire et d'agro-industries rurales, qui semblent « faire système ».

6 Les Syal sont alors définis comme "des organisations de production et services (unités de production agricole, entreprises agro-alimentaires, commerciales, de service, restauration) associées de par leurs caractéristiques et leur fonctionnement à un territoire spécifique. Le milieu, les produits, les hommes, leurs institutions, leur savoir-faire, leurs réseaux de relations, se combinent dans un territoire pour produire une forme d'organisation agro-alimentaire à une échelle spatiale donnée » (Fourcade, 2008 : 194 ; Cirad-Sar : 1996).

7 Le Syal recouvre donc, en premier lieu, une dimension organisationnelle relative à la constitution de réseaux localisés d'entreprises, qui permet aisément d'assimiler les Syal à «des SPL spécifiques dans le domaine agroalimentaire ( (Fourcade, 2006a : 185). Le Syal peut également faire l'objet d'une "approche produit» et/ou d'une «approche environnement ». L'approche produit permet d'analyser les liens entre territoire, qualité, et habitus alimentaires ou de consommation, très spécifiques aux filières agroalimentaires (Fourcade et al., 2010). L'approche environnement scelle quant à elle l'ancrage territorial du Syal autour des problématiques liées à la multifonctionnalité de l'agriculture et à 
l'usage des ressources naturelles locales, en lien avec certains enjeux du développement durable (Rodriguez-Borray et Requier-Desjardins, 2005 ; Requier-Desjardins, 2010).

Selon Fourcade et al. (2010), deux perspectives de recherche émergent désormais du foisonnement des travaux portant sur cet objet. Si la première fait du Syal un outil pour l'analyse des différentes formes d'organisations localisées de l'agroalimentaire, la seconde, dans laquelle se situe notre analyse, fait du Syal un «levier stratégique » pour la définition de stratégies de développement local (Boucher et al. 2010). Dans cette perspective, dite "stratégique », les Syal sont alors appréhendés comme des "modèles de développement agroalimentaires fondés sur la mise en valeur des ressources locales, plus respectueux de l'environnement, plus attentifs à la qualité et à la diversité de produits agricoles et alimentaires, plus soucieux des dynamiques locales de développement et de nouveaux enjeux du monde rural » (Muchnik, $2002: 3$ ). Le recours au concept de stratégie collective - lui-même emprunté ici au champ disciplinaire de «l'écologie humaine " (Astley et Fombrun, 1983) -, permet ensuite d'expliquer «les formes collectives et proactives de l'adaptation organisationnelle à l'environnement » (Yami, 2006 : 92). L'analyse des Syal débouche alors sur un postulat selon lequel, les communautés rurales et, plus particulièrement, les paysans, témoignent d'une propension à coopérer et à développer des formes organisationnelles innovantes afin de s'adapter à un environnement turbulent en réduisant collectivement leur vulnérabilité individuelle. Effectivement, la dépendance envers les ressources naturelles locales, les conflits d'usages de ces mêmes ressources - l'eau par exemple - la vulnérabilité face au changement climatique, ou encore la vulnérabilité face à la volatilité des prix sur les marchés agricoles, comme la concurrence des grandes multinationales de l'agroalimentaire, constituent autant d'éléments qui favorisent l'émergence de comportements coopératifs entre agents des territoires ruraux (Fourcade et al., 2010; Larroa, 2010).

9 Cette perspective de recherche rencontre un écho particulier dans le contexte des pays en développement, notamment en Amérique Latine, là où les travaux sur les Syal trouvent leurs origines. Comme le souligne Larroa (2010), dans le cas particulier de l'Amérique Latine persiste une population paysanne, pauvre, minifundiaire et qui, le plus souvent, maintient très vives les caractéristiques culturelles liées à ses origines ethniques. L'attachement et le sentiment d'appartenance à un système de valeurs différent de celui véhiculé par l'idéologie néolibérale ne fait ainsi que renforcer la propension à agir collectivement dans les zones rurales pauvres d'Amérique Latine (cf. les travaux de la Via Campesina sur la Souveraineté Alimentaire ; Figuière et Metereau, 2012).

Dans ce contexte, «le Syal est un outil méthodologique qui peut être mis à la disposition des paysans et des communautés rurales qui s'organisent dans leur combat pour résister et lutter contre l'exclusion sociale dont ils font l'objet depuis l'avènement de la mondialisation néo-libérale en Amérique latine, sans avoir à renoncer à leur culture et sans perdre leurs ressources foncières ${ }^{3}$ (Larroa, 2010 : 1).

11 Le Syal pourrait donc constituer un "outil méthodologique " permettant la définition de projets de développement rural local, reposant sur la valorisation des ressources territoriales, le respect des cultures, la prise en compte des enjeux spécifiques au monde rural, voire, une meilleure intégration de la dimension environnementale et des défis relatifs à la durabilité du développement.

12 Ces « méthodes Syal », nécessairement hétérogènes du fait de la diversité des trajectoires de développement local et des modes de coordinations que peut recouvrir la notion de Syal (Fourcade, 2006b), auraient donc pour objectif central de renforcer la capacité 
d'action collective des acteurs du territoire. Fournier et Muchnik (2010:12-13) suggèrent pour cela quatre axes d'action: la capacitation des organisations locales (notamment organisations de producteurs) ; l'affirmation des spécificités du territoire et des produits, des démarches collectives de qualification, ou encore, le renforcement des complémentarités entre la filière motrice et les autres activités du territoire.

Il convient désormais de préciser les modalités d'une intégration plus formelle de la dimension environnementale à ce type de démarche.

\section{Système Agroalimentaire Localisé : la durabilité en question.}

On prête en effet volontiers au Syal une capacité à intégrer la contrainte environnementale dans l'élaboration d'une stratégie de développement local. Cette "durabilité » supposée, évoquée comme l'un des avantages de ce type de coopération localisée dans le secteur agroalimentaire, repose sur un postulat, que certes nous partageons, mais qui ne suffit pas à garantir au Syal cette capacité qui lui serait intrinsèque.

Deux types d'arguments, chacun lié aux particularismes du secteur agricole, sont mobilisés pour justifier cette prise en compte de la dimension environnementale :

- La multifonctionnalité de l'agriculture est censée se transférer au niveau de l'organisation. Au-delà de la production agricole et de la fourniture d'aliments, l'agriculture offre également un certain nombre de services environnementaux ou écologiques (Mollard, 2003), voire même sociaux (Rodriguez-Borray et RequierDesjardins, 2005). Dans le cadre du Syal, cette multifonctionnalité de l'agriculture qui reposait sur les pratiques individuelles des agriculteurs, est donc censée se transférer à l'échelle de l'organisation, en supposant donc la prise en compte d'enjeux environnementaux et sociaux dans l'élaboration des stratégies collectives des acteurs de la filière agroalimentaire ;

17 - La relation particulière de grande dépendance qui lie le secteur agricole à son environnement naturel et la disponibilité de ses ressources renforce de facto la mise en relation entre les enjeux du Syal et ceux de la durabilité du développement (RequierDesjardins, 2010). Le Syal permettrait ainsi la mise en œuvre d'actions collectives visant à la préservation d'un « patrimoine naturel local » indispensable au maintien de l'activité agricole. L'affirmation des spécificités du territoire et des produits, leur qualification, passe généralement aussi par la préservation d'un environnement naturel particulier. $\mathrm{Ce}$ type d'action collective peut également s'inscrire dans la cadre de phénomènes d'adaptation au changement climatique qui nécessitent une évolution des pratiques, la diffusion de nouvelles connaissances, voire l'introduction de nouveaux types de culture (exemple de l'agroforesterie et de la culture du cacao dans la région frontalière du Rio San Juan au Nicaragua) ${ }^{4}$.

Il existe donc effectivement plusieurs raisons de considérer que le Syal a un rôle à jouer en matière de durabilité du développement, particulièrement en ce qui concerne le volet environnemental. Néanmoins, ce qui peut être postulé à partir de ces éléments, c'est une propension à prendre en compte la dimension environnementale du fait du statut effectivement particulier du secteur agroalimentaire. Cela ne signifie pas pour autant que le Syal, en l'état actuel des propositions, constitue un "outil méthodologique» pour 
l'intégration de cette dimension dans des stratégies de développement localisé. L'intensité des relations, la multiplication des comportements de coopération au sein d'un espace localisé, conduisent l'organisation socio-économique à faire système. Dès lors, ces processus peuvent favoriser l'émergence d'actions collectives, qui, du fait des particularismes $\mathrm{du}$ secteur agricole précédemment mentionnés, sont propices à l'intégration d'une dimension environnementale.

S'il y a donc un « support » favorable à cette intégration, on ne peut faire l'économie de penser une méthode, des outils, des principes opérationnels qui permettraient de programmer cette intégration à moyen et long terme et ses impacts sur la structure et l'organisation socio-économique des Syal. L'agroécologie, comme méthode de culture, et l'IFES (Integrated Food Energy System), comme principe de complémentarité entre production agricole (pour l'alimentation) et production énergétique, constituent une première base opérationnelle pour des organisations « écosystémiques ».

\section{L'agroécologie et l'IFES comme premiers « compléments environnementaux » du Syal.}

20 La base agricole du Syal va nous guider vers les outils et les méthodes qui permettent de renforcer la durabilité environnementale du système agroalimentaire. Notre choix se restreint ici à deux d'entre eux, considérés comme compatibles et très prometteurs en termes de durabilité environnementale : l'agroécologie et l'IFES.

21 L'agroécologie vise au design et à la gestion durable des agroécosystèmes. Pour cela, sont promues des pratiques alternatives - polyculture, permaculture, agroforesterie etc. - qui permettent d'obtenir des rendements au moins équivalents à ceux d'une agriculture conventionnelle en monoculture, tout en réduisant leur impact sur l'environnement. Cet objectif ambitieux prend appui sur deux axes :

22 - La substitution des intrants biochimiques et énergétiques nécessaires à l'agriculture conventionnelle par une meilleure appréhension, interactions et synergies entre les composants d'un écosystème. Ce sont en effet ces mécanismes qui permettent de fournir un certain nombre de services écologiques utiles à la productivité agricole (fertilisation, lutte contre l'érosion, utilisation plus efficace de la ressource en eau, réduction des pertes liées aux plantes, insectes et maladies nuisibles aux récoltes) (Altieri et Nicholls, 2008 ; Altieri, 2009);

23 - Un double adossement, entre savoir paysan et savoir scientifique, pour parvenir à une meilleure appréhension des écosystèmes naturels, ainsi qu'à un design et une gestion plus efficients de l'agroécosystème. Il s'agit de l'association entre, d'une part, les savoirs traditionnels issus d'une démarche "paysans à paysans » (partage des savoirs et savoirfaire et action collective) (Holt Giménez, 2008) pour la compréhension des phénomènes et particularismes locaux, et, d'autre part, les apports de la science pour l'étude et la compréhension plus fondamentale et transversale des systèmes biologiques, comme des flux et stocks de matières et d'énergies (Altieri, 2002 ; Altieri et Nicholls, 2005 ; Gliessman, 2007).

24 Il s'agit bien ici de promouvoir une pratique localisée, durable et largement favorable au renforcement des capacités des communautés rurales et de leur autonomie. Dans le cadre de l'agroécologie, des "small integrated farming systems" (Altieri et Nicholls, 2008 : 474) sont implémentés. Ils reposent sur l'identification et le recours à toutes les complémentarités 
potentiellement bénéfiques entre les diverses activités de production et de consommation de l'agroécosystème.

Le projet d'"Integrated Food-Energy Systems" (IFES) (Sachs et Silks, 1990 ; Bogdanski et al., 2011) 5 peut, quant à lui, être interprété ex post (les travaux sur l'IFES se développent à peu près sur la période où émergent les premières propositions sur l'agroécologie) comme un " approfondissement thématisé » de celui de "small integrated farming systems" en réalisant un focus sur l'imbrication entre production d'aliments et production d'énergie. L'IFES met ainsi en exergue le potentiel d'autonomie que peut générer la satisfaction conjointe de ces deux besoins essentiels.

En effet, l'IFES consiste à transposer les modes d'organisation écologiques basés sur le bouclage des flux (closed-loop) (Sachs et Silk, 1990) de façon à intégrer, intensifier et accroitre la production d'aliments et d'énergie en transformant les sous-produits agricoles en ressources (fertilisants et énergie). Deux types d'IFES sont distingués dans les travaux.

Les IFES de type 1 "are characterized through the production of feedstock for food and for energy on the same land, through multiple-cropping patterns or agroforestry systems" (Bogdanski et al., 2011:5).

Effectivement, en agroécologie, la productivité par surface cultivée ne se limite pas au rendement obtenu avec une seule espèce. Sur la même parcelle, d'autres productions peuvent être valorisées, notamment de la matière première (biomasse) pouvant à la fois servir d'engrais ou de source d'énergie. Dans le cas d'une pratique en agroforesterie, les arbres, partie intégrante et contributive du fonctionnement de l'agroécosystème (Service écosystémique), fournissent également de la matière première, non seulement alimentaire (fruits) mais aussi pour le chauffage, la construction, la cuisine etc. (Service productif).

Les IFES de type 2 "seek to maximize synergies between food crops, livestock, fish production and sources of renewable energy. This is achieved by the adoption of agro-industrial technology (such as gasification or anaerobic digestion) that allows maximum utilization of all by-products, and encourages recycling and economic utilization of residues." (Bogdanski et al., 2011 : 5).

Dans cette seconde option, une composante technologique est intégrée au déploiement d'une organisation circulaire de la production à l'échelle de l'agroécosystème. La méthanisation, au moyen de la mise en œuvre de biodigesteurs, devient ainsi une clé du bouclage des flux de matières et d'énergie au sein de la ferme (Michel et al., 2010), ou de la coopérative, en permettant l'utilisation des résidus de production, par exemple la bagasse issue de la production de canne à sucre (Ometto et al., 2004). On permet ainsi la production conjointe, non-concurrentielle (conflit d'usage des terres entre, d'une part, la production d'aliment, et, d'autre part, la production de matières premières/énergie), et autonome d'aliments et d'énergie, considérés dans le projet comme les deux clés de la réduction de la pauvreté.

31 Le couple agroécologie/IFES permet ainsi d'envisager la concrétisation d'une organisation durable des activités de production et de consommation à l'échelle micro-socioéconomique. Il peut être vu comme un embryon d'organisation écosytémique de la production, plus ou moins complexe et, a minima, aisément assimilable à une tentative de bouclage des flux de matières et d'énergie telle que l'EI propose de l'implémenter à l'échelle des territoires. "L'IFES agroécologique » constitue ainsi une synergie de base, un "sous-système ", qui peut être intégré à un ensemble systémique plus large et plus 
complexe, " plus anthropisé ». Cette combinaison est donc à la fois synergie de base et interface direct entre écosystèmes naturels et systèmes anthropiques (Gomiero et al., 2006). Cette amorce d'organisation écosystémique dans le secteur agricole, d'ores et déjà opérationnelle (Pretty et al., 2003 ; Altieri et al., 2012 ; Ometto et al., 2004), motive alors la recherche d'une voie de généralisation de l'approche écosystémique des organisations socio-économiques. La recherche des complémentarités et du bouclage des flux de matières et d'énergie deviendrait ainsi l'un des déterminants majeurs dans l'élaboration de stratégies de développement local durable en milieu rural. Dans cette perspective, l'apport de l'écologie industrielle peut s'avérer substantiel pour systématiser et généraliser le principe de bouclage des flux de matières et d'énergie esquissé par les outils déjà mobilisés.

\section{Le bouclage des flux comme principe fondateur de l'écologie industrielle.}

32 Très sommairement on peut résumer la proposition centrale de l'écologie industrielle par le principe de bouclage des flux, ce principe étant considéré ici comme un moyen particulièrement prometteur d'internaliser la préoccupation environnementale dans l'organisation des activités humaines. Parmi les travaux sur l'écologie industrielle, nous allons distinguer trois catégories.

Les premiers sont ceux qui fondent le socle de l'écologie industrielle en détaillant les outils à mettre en œuvre et les principes qui les guident: limiter l'impact global de l'activité humaine sur son environnement naturel en bouclant les flux d'énergie et de matières. Ces travaux sont issus de différentes disciplines, notamment des écologues mais aussi de diverses branches des sciences de l'ingénieur. Ils sont à l'origine de la proposition et continuent à se développer.

es deux autres catégories de travaux distinguées ici portent sur les modalités de l'appropriation de ce socle. Ces propositions sont portées par des chercheurs issus de différentes disciplines, des économistes notamment. La deuxième catégorie va donc regrouper les réflexions qui vont s'inscrire dans une démarche qualifiée "d'objective ", mais qui se situent de facto, bien qu'implicitement, dans une approche que l'on qualifie ici, en accord avec Opoku et Keitsch (2006) de « libérale » et en durabilité faible. La troisième catégorie regroupe les travaux qui abordent la mise en place l'EI en adoptant une démarche explicitement théorique, normative et qualitative. Ces travaux peuvent être situés en socio-économie politique, ils affichent leur hétérodoxie et leur positionnement en durabilité forte.

Avant toute chose, il convient ici de rappeler que la distinction entre les versions faible et forte de la durabilité, se fonde sur des critères économiques. En effet, en partant de la proposition faite par Daly (1991), la durabilité forte se distingue par les trois critères suivants: faible substituabilité entre capital «créé par les hommes» et "patrimoine naturel»; seuils d'irréversibilité; valeur des biens environnementaux non nécessairement « monétarisable ».

Si les travaux se situant dans la troisième catégorie sont facilement identifiables, il est beaucoup moins aisé d'établir une frontière claire entre les deux premières catégories.

Ainsi si les écrits de Frosh et Gallopoulos (1989) ou de Erkman (1998) peuvent assez aisément être considérés comme portant sur les fondamentaux de l'EI, les propositions 
faites par un auteur comme Allenby vont devoir faire l'objet d'un examen beaucoup plus fin: certaines des plus anciennes peuvent sans conteste faire partie de la première catégorie (Allenby et Cooper, 1994), d'autres, plus récentes (Allenby, 1999a, 1999b), peuvent au contraire être considérées comme se situant dans l'approche libérale sur l'EI.

Le graphique suivant permet de positionner ces trois catégories de travaux : l'une d'entre elle constituant le point de départ commun pour les deux autres. L'analyse qui est menée ici se situe dans la vision dite « socio-économique » de l'EI.

Figure 1 : Trois catégories de travaux en Écologie Industrielle

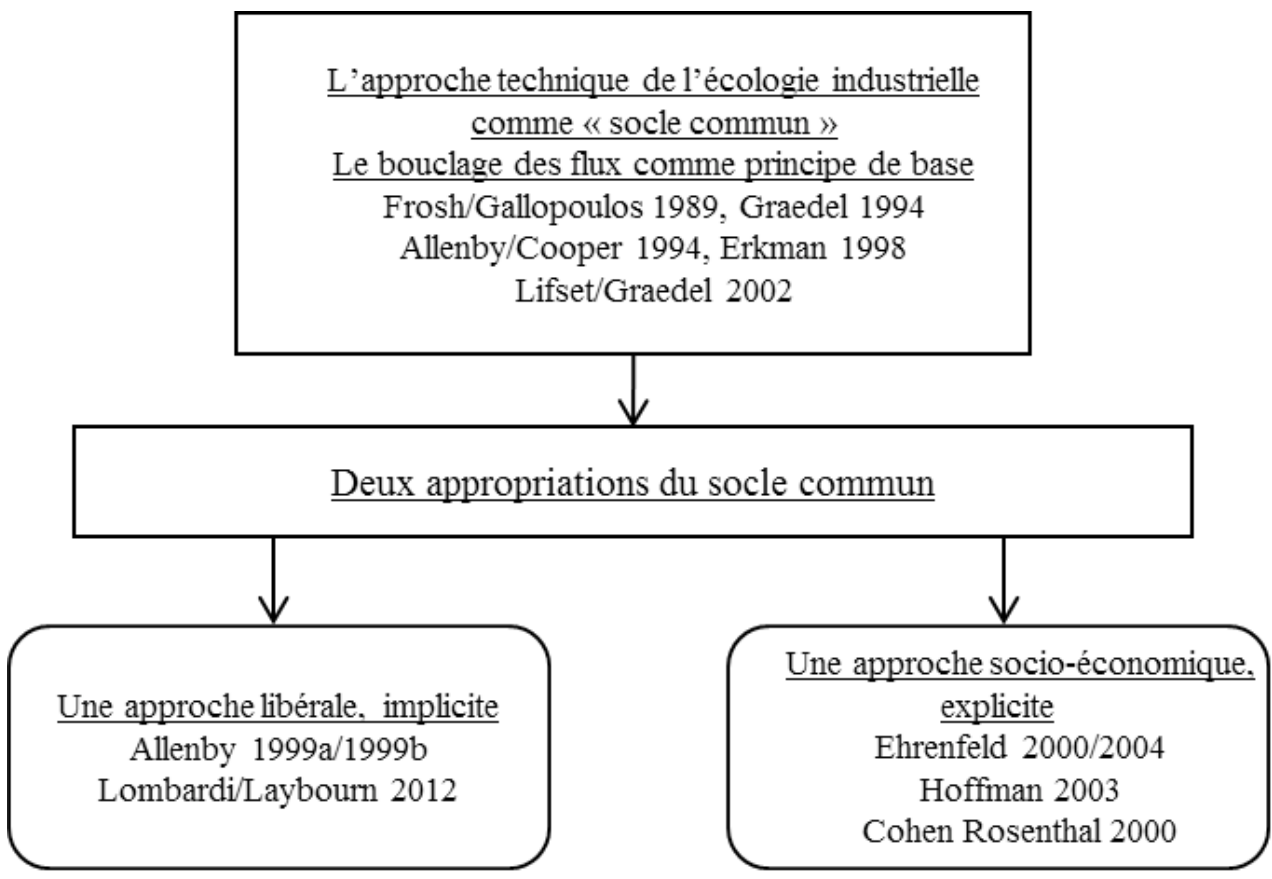

Les travaux du "socle commun ", ou "approche technique ", procèdent par analogie entre les écosystèmes naturels et les systèmes industriels (Graedel et Allenby, 1995). Le terme "écologie» fait référence à l'étude scientifique des écosystèmes. Le terme « industrielle », ne restreint pas la démarche à un secteur d'activité, mais désigne l'ensemble des structures anthropiques «modernes » (société industrielle) consommant, échangeant ou rejetant de la matière ou de l'énergie (Jelinski et al., 1992). Il s'agit ainsi d'appliquer les méthodes d'analyse des métabolismes biologiques à l'étude du «métabolisme industriel». Suren Erkman, pionnier de la divulgation de l'EI dans la littérature francophone, définit l'étude du métabolisme industriel comme suit :

"L'étude des ensembles des composants biophysiques du système industriel. Cette démarche, essentiellement analytique et descriptive, vise à comprendre la dynamique des flux et des stocks de matière et d'énergie liées aux activités humaines, depuis l'extraction et la production des ressources jusqu'à leur retour inévitable, tôt ou tard, dans les processus biogéochimiques " (Erkman, 1998 : 10).

41 L'analyse approfondie du métabolisme industriel doit permettre d'identifier des synergies potentielles entre les différentes composantes du système industriel, afin de réaliser des « symbioses industrielles ». C'est ainsi que l'EI peut contribuer à la réduction de l'impact des activités humaines sur la biosphère - éco-efficience - et, de manière plus ambitieuse, en tenant compte des interactions entre les sociétés humaines et la nature, amorcer la 
réintégration des systèmes anthropiques au fonctionnement normal de la biosphère. Une démarche d'EI vise donc à entraîner la transition d'un système linéaire (écosystème "juvénile», ou de type 1) et de traitement "end of pipe" des pollutions (Erkman, 1998), "dilapidaire» des ressources physiques et énergétiques, à un système "circulaire" (Mathews et Tan, 2011) ou «bouclé », plus économe et donc plus efficient (écosystème «mature », ou de type 3)

La typologie proposée par Graedel (1994) exprime cette analogie évolutive d'un système simple à un système complexe. Néanmoins, un fonctionnement du système industriel semblable à celui d'un écosystème "mature", de type 3 , reste un objectif difficile à atteindre. Un objectif plus réaliste consiste à se rapprocher d'un « écosystème industriel idéal » dont les besoins en ressources et les rejets sont fortement limités grâce notamment à une amorce de bouclage des flux (flux quasi-circulaires de matières et d'énergie : écosystème de type 2).

43 Concrètement donc, l'EI vise au redesign des systèmes productifs à partir d'une analyse fine du métabolisme industriel permettant d'identifier les interrelations potentielles afin de boucler le plus de flux de matières et d'énergie possibles.

\section{L'approche socio-économique de l'El : « levier stratégique » de la durabilité du développement local}

Le socle commun - l'analogie avec les écosystèmes naturels et la recherche du bouclage des flux de matières et d'énergie -, partagé par l'ensemble de la communauté de chercheurs et d'ingénieurs-techniciens de l'écologie industrielle vient d'être précisé. Nonobstant, dans la pratique, la transposition des enseignements de l'écologie scientifique ne se fait pas toujours de manière aussi rigoureuse que l'analogie fondatrice de l'EI le suggère. Elle donne lieu à des appropriations différentes (Hess, 2009), notamment dans le champ des sciences sociales qui sont nécessairement mobilisées lorsqu'il s'agit d'appliquer l'EI à des ensembles socio-économiques (Allenby, 1999b). Un clivage apparait donc quant au statut et à la portée effective de l'écologie industrielle. Plus fréquemment appréhendée du point de vue des sciences sociales comme une science neutre au service de l'éco-efficience, on peut également voir dans l'EI, la proposition d'une évolution en profondeur de nos sociétés, ce qui va de fait lui conférer un caractère « intrinsèquement normatif » (Boons et Roome, 2000).

Nous retrouvons dès lors les deux approches établies dans le point précédent. Dans la première, qualifiée ici de "libérale ", les déterminants sociaux et culturels de la mise en œuvre de ces évolutions techniques majeures doivent être intégrés aux réflexions en termes d'EI comme des données exogènes, via des indicateurs «objectifs" (Allenby, 1999b). Opoku et Keitsch (2006) remettent largement en question cette "objectivité » affichée, en soulignant la forte connotation des postulats implicites, fondements de cette approche : le « déterminisme technologique $»^{7}$ et le « libéralisme traditionnel ». L'efficience des marchés et le progrès technique sont dès lors censés mener les acteurs économiques à multiplier les échanges de matières et d'énergie, devenus à la fois économiquement viables et environnementalement bénéfiques (Lombardi et Laybourn, 2012). On se situe alors dans la perspective « gagnant-gagnant » de l'éco-efficience (Deutz et Gibbs, 2008).

Cette approche, aboutissant au verdissement des activités économiques dans un contexte "business as usual", entérine implicitement les mécanismes de marché et du progrès 
technique (Allenby, 1999a) comme les normes sur lesquelles se fondent les démarches d'EI. L'EI n'est dès lors pas envisagée comme une démarche plus proactive et interventionniste susceptible de répondre aux évolutions profondes que suggère l'impératif de durabilité (Wells et Zapata, 2012).

La seconde approche, "socio-économique ", s'affiche comme théorique, normative et transdisciplinaire (Sachs, 1980, 1990 ; Ehrenfeld, 1997 ; Cohen-Rosenthal, 2000). L'intérêt se porte en premier lieu sur les "caractéristiques structurelles et organisationnelles de l'analogie avec les écosystèmes naturels " (Beaurain et Brullot, 2011 : 317). La mise en œuvre de l'EI ne peut dès lors se faire qu'au moyen de changements radicaux dans la structure, l'organisation et les institutions de nos sociétés (Hill, 2006). Cette démarche suppose d'appréhender le système productif comme un système ouvert, en interaction complexe avec l'ensemble des composants d'une société (Hoffman, 2003). Dans ce contexte, le bouclage des flux de matière et d'énergie au sein des systèmes industriels est autant un problème relevant des structures socio-économiques qu'un problème technique, ou relevant d'une simple analyse coût/bénéfice de l'éco-efficience. Les dimensions sociales, politiques et culturelles de la transformation du système industriel deviennent ainsi prédominantes dans la mise en œuvre de l'EI, qui relève en outre d'une approche en «durabilité forte ».

48 L'EI constitue dès lors un nouveau paradigme dans le champ du développement et de sa durabilité (Ehrenfeld, 2000), inspiré des structures et du fonctionnement des systèmes biologiques.

"The best route toward sustainability can be mapped by replacing the elements of the modernist social paradigm with a new set mimicking nature, bringing industrial ecology back to its foundation in the biological metaphor." (Ehrenfeld, $2007: 82$ ).

50 Cette "vision" normative de ce que devraient être des systèmes industriels durables, inspirée de la métaphore de l'écosystème naturel (Hess, 2009; Ehrenfeld, 2007), fait émerger deux principales caractéristiques d'une approche « socio-économique » de l'EI :

51 - Une démarche holistique (Hoffman, 2003). L'élargissement du champ de l'EI, au-delà de la concrétisation du principe de bouclage des flux de matières et d'énergie restreinte à des petits groupes d'acteurs (souvent des producteurs), pour s'intéresser également aux déterminants sociaux, politiques et culturels de la systématisation de ce principe, place d'emblée cette approche dans une démarche holistique. L'EI vise ainsi à appréhender les systèmes sociaux économiques et industriels comme des systèmes complexes. On ne s'intéresse pas aux acteurs individuels (ou à des agrégations d'acteurs individuels) mais à des « communautés» d'acteurs (Bey, 2001) faisant système à une échelle spatiale donnée. On se concentre donc sur les aspects structurels et organisationnels de la métaphore de l'écosystème, et sur les conditions de la généralisation du principe de bouclage des flux au-delà de quelques symbioses isolées. Pour cela, le rôle des institutions, la nature et l'intensité des réseaux sociaux, des interactions formelles et informelles, marchandes et non-marchandes, entre acteurs, deviennent des éléments centraux pour la concrétisation des démarches d'EI (Ashton, 2008; Hoffman, 2003). Cette dernière peut dès lors s'inscrire dans la lignée de certains travaux portant sur les réseaux d'entreprises (Cohen Rosenthal, 2000), qui mettent en avant les externalités positives attribuables à des formes variées de proximités (Bouba-Olga et Grossetti, 2008).

52 - Une approche territoriale ou localisée. Autant d'un point d'un vue théorique que pratique, une démarche d'écologie industrielle "socio-économique», convoque une 
approche territoriale. D'abord du point de vue théorique (épistémique), la métaphore de l'écosystème indique clairement la prise en compte de systèmes industriels localisés ; c'est-à-dire appartenant à un ensemble physique et culturel délimité, à l'image de l'écosystème naturel, réunissant un ensemble d'êtres vivants (biocénose) au sein d'un environnement particulier (biotope) qui en délimite les frontières. En termes pratiques ensuite, deux voies permettent de justifier le caractère nécessairement localisé d'une démarche d'EI. Du point de vue du développement durable, qu'il s'agit ici d'opérationnaliser, les problèmes sont souvent posés à l'échelle globale. L'espace des réponses, lui, se situe généralement plutôt à l'échelle locale (Vivien et Zuindeau, 2001 ; Theys, 2002 ; Godard, 2005). La mise en œuvre de l'EI quant à elle, consiste à repérer et à concrétiser des synergies potentielles entre des acteurs géographiquement proches (Ashton, 2009; Buclet, 2011) révélant ainsi, de fait, une dimension territoriale (Beaurain et Brullot, 2011; Maillefert et Schalchli, 2012), et, laissant entrevoir une perspective en termes de stratégie de développement local (Deutz et Gibbs, 2008)9 .

\section{Au carrefour du Syal et de l'El..., éléments de bouclage}

l'EI est désormais lue comme un «levier stratégique » en matière de durabilité. Cette approche «socioéconomique » de l'EI s'avère en effet complémentaire des approches en termes de système socio-économique localisé. Dans les deux cas, le focus porte sur des systèmes d'acteurs localisés (communautés), la mise en relation et les comportements coopératifs de ces acteurs générant potentiellement des externalités positives, de nature socioéconomique dans le cas du Syal, de nature environnementale dans le cas de l'EI. En fournissant un nouveau cadre paradigmatique et des outils méthodologiques appropriés, l'EI pourrait ainsi constituer une voie d'intégration de la dimension environnementale au sein de systèmes socio-économiques localisés.

La pertinence d'une combinaison entre le principe de bouclage des flux de l'EI d'une part, et, du Syal d'autre part, est en outre renforcée par l'existence des prémisses d'une organisation socio-économique écosystémique à partir du secteur agricole, sur la base notamment de la diffusion de l'agroécologie et plus particulièrement de l'implémentation d'IFES. Ce dernier projet permettant de rendre opérationnelle une synergie entre production d'aliments et production d'énergie à partir d'une ressource renouvelable : la biomasse issue des sous-produits agricoles. Ci-dessous, le tableau 1 synthétise les caractéristiques des quatre pièces de la combinaison proposée par cet article. Elles sont ensuite agencées dans la figure 2 . 
Tableau 1 : Les éléments constitutifs du DRLD

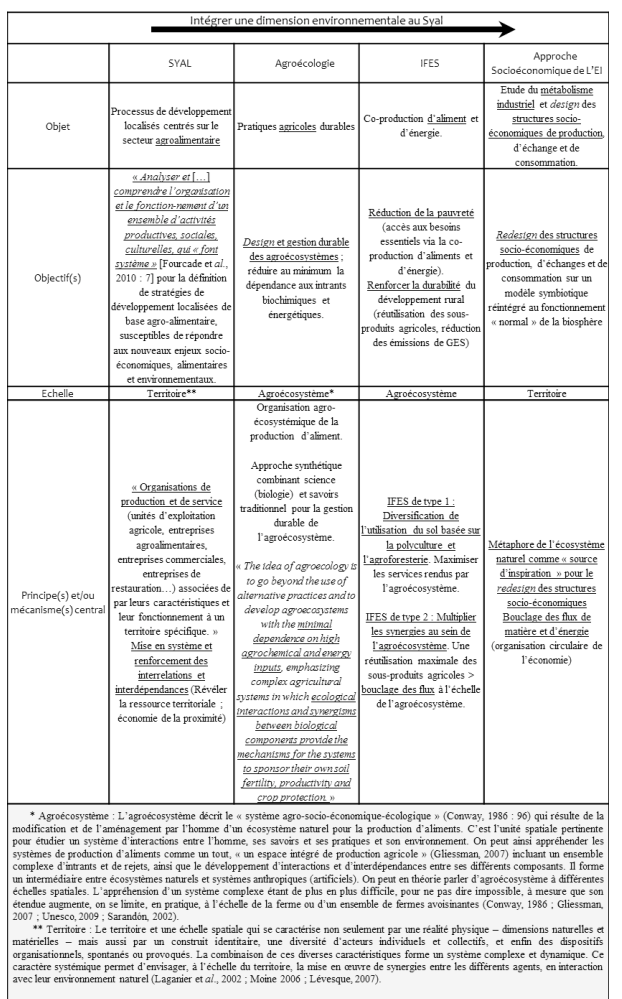

figure 2 : Premières propositions pour un développement rural local durable DRLD

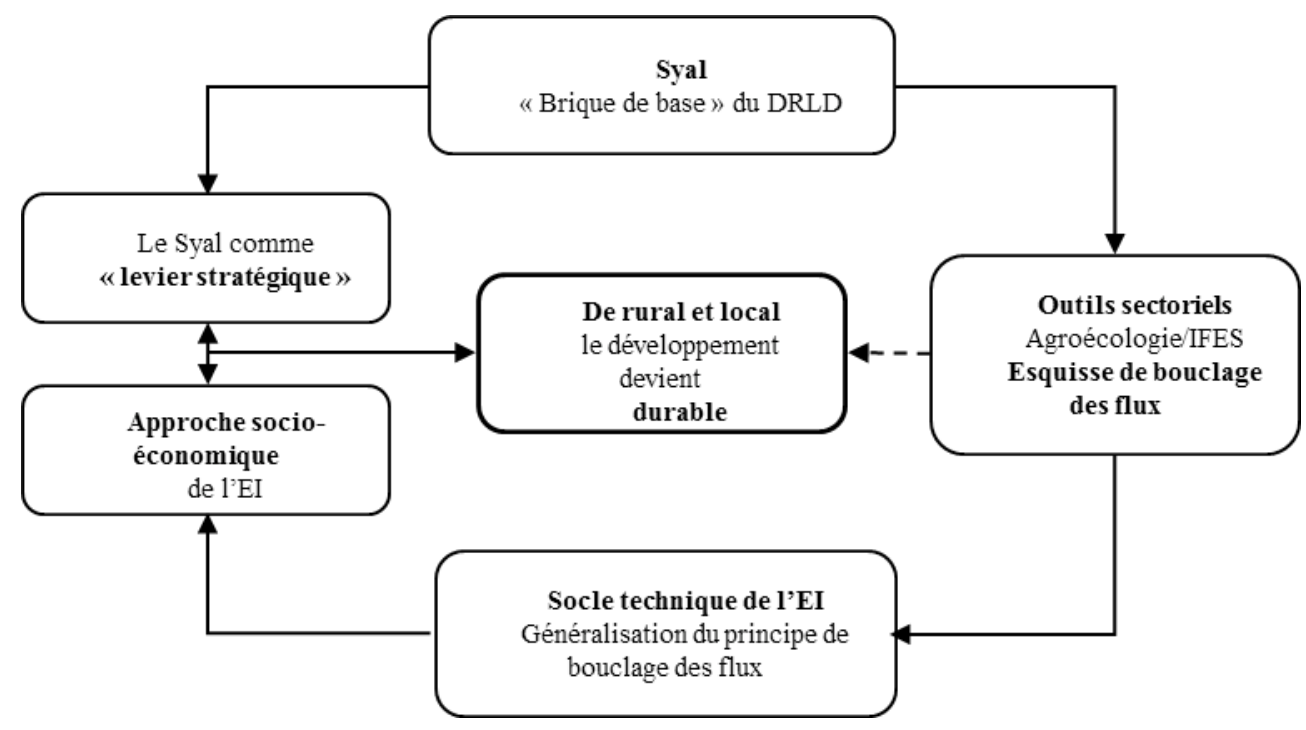

Il convient désormais de proposer des éléments de réflexion qui permettraient de dépasser les difficultés soulevées par cette combinaison. La mise en œuvre de l'EI comme une stratégie de développement local implique en effet que des réseaux d'acteurs se constituent, ou se renforcent, autour d'un objectif d'ordre environnemental dont les bénéfices économiques sont, au moins à cours termes, incertains. C'est cette caractéristique qui, en premier lieu, différencie les démarches « d'écologie industrielle et territoriale ", d'autres types de stratégies de développement local basées sur la création de réseaux d'interrelations et d'interdépendances telles que le cluster, le SPL, ou encore 
le Syal (Deutz et Gibbs, 2008). Deutz et Gibbs identifient une seconde difficulté: les démarches éco-industrielles se basent généralement sur des réseaux interentreprises constitués ex-nihilo. Cette seconde caractéristique conjuguée à la première rend très ambitieuse toute stratégie visant à implémenter l'écologie industrielle comme une stratégie de développement local. Le seul objectif environnemental reposant sur le bouclage des flux de matières et d'énergie ne suffit plus, dans ces conditions, à faire émerger les interdépendances et l'ancrage territorial nécessaires au succès de ce type de stratégie. Ce sont alors d'autres motifs de coopération, principalement de nature économique (mutualisation de services, économie d'échelle, réduction des coûts de transaction etc.), qui deviennent déterminants.

Dès lors, mettre en œuvre des démarches d'écologie industrielle à partir d'un Syal, c'està-dire à partir de réseaux d'acteurs existants et déjà fortement ancrés au territoire, pourrait apporter un élément de réponse à ce type de difficulté. Il s'agirait alors d'amorcer un bouclage des flux de matières et d'énergie en partant de l'existant, par l'étude du métabolisme territorial de référence et l'identification de synergies potentielles. C'est sur cette base qu'il serait possible dans un second temps, de prendre en compte des critères éco-industriels pour l'intégration de nouvelles activités à un système socio-économique localisé déjà pérenne.

Cette mise en œuvre, à partir d'un Syal existant, introduit ce faisant une contrainte supplémentaire en imposant les "espèces »/activités présentes dans "l'écosystème industriel ». Dans le cas du Syal en effet, la constitution d'un réseau d'entreprises se fait généralement autour d'une filière motrice et souvent autour d'agro-industries rurales de transformation qui permettent de mieux valoriser la production paysanne (Boucher et al. 2003 ; Boucher, 2012). Cette «spécialisation » reste néanmoins relative. Si la constitution du Syal, autour de l'intensification des comportements de coopération, se concrétise grâce à l'essor d'une filière motrice, cela ne signifie pas pour autant la disparition des autres activités du territoire. À l'inverse, les processus de qualification par le territoire prennent appui, dans le cas des démarches de type "panier de biens » (Pecqueur, 2001; Mollard et Pecqueur, 2007), sur la complémentarité entre filières agricoles et diversité des activités locales, d'artisanat ou de tourisme par exemple (Fournier et Muchnik, 2010). L'une des spécificités des Syal vis-à-vis des SPL ou des Cluster serait alors leur «pluriactivité » (Resquier-Desjardins, 2007). Au lieu d'une spécialisation a priori défavorable au bouclage des flux de matières et d'énergie, le Syal peut donc révéler un système diversifié à même de faire apparaitre des synergies éco-industrielles potentielles entre, notamment, une « espèce clé» (Erkman, 1998) (la filière motrice) et les autres activités du territoire. En termes concrets, la spécialisation et l'intensification des coopérations à l'échelle locale dans la région du Rio San Juan au Sud-Est du Nicaragua, centrées sur l'émergence d'une filière agro-exportatrice de cacao bio-équitable, se fait de concert avec le maintien d'autres types de cultures (agriculture vivrière et/ou destinée aux marchés locaux), et le développement d'activités connexes (éco-tourisme, artisanat, transport etc.). Si l'intensification des comportements de coopération constitutifs d'un Syal se réalise à partir de l'essor d'une filière motrice, cela correspond éventuellement à une "spécialisation » de la coopération, mais pas du territoire en lui-même. Le potentiel écoindustriel en termes de synergies reste donc entier. Il s'agit alors d'étendre le champ de la coopération à des thématiques environnementales qui peuvent amener à transcender «la logique filière ». 
Enfin, les Syal sont des objets de recherche très hétérogènes de par la diversité des compositions territoriales, des trajectoires de développement et des modes de coordination (Fournier et Muchnik, 2010). Il ne serait donc certainement pas opportun de vouloir fixer les « règles » d'une telle combinaison entre Syal et principe de bouclage des flux de l'EI. Nonobstant, Fourcade et al. (2010) ouvrent une perspective intéressante dans le contexte français, en proposant une typologie en quatre catégories des formes de coopérations localisées entre entreprises de l'agroalimentaire. La prise en compte de la dimension environnementale comme élément déterminant de la structure du système productif local, tel que le suggérerait la mise en œuvre de l'EI à l'échelle d'un Syal, pourrait être conditionnée par la forme de stratégie collective déjà à l'œuvre dans ledit Syal. Ainsi certaines formes de stratégies collectives - a priori celles correspondant à des formes d'associations dites "symbiotiques» (Yami, 2006) - seraient plus à même que d'autres, de « supporter » une démarche éco-industrielle.

\section{Conclusion}

59 Finalement, la systématisation du type de démarche porté par l'écologie industrielle boucler les flux de matières et d'énergie au sein d'un système socio-économique localisé pourrait contribuer à renforcer la durabilité des Syal. Cela rendrait possible la définition, au cas par cas, de stratégies de Développement Rural Local Durable en intégrant l'objectif de bouclage de flux aux stratégies collectives préalablement élaborées dans la cadre d'une démarche de type Syal. Cette intuition, pour être validée, nécessite néanmoins de développer a minima deux pistes de recherche :

60 - D'abord, il sera nécessaire d'étudier la possibilité d'une concordance, en termes de complémentarités et de coordination des acteurs, entre d'une part la « logique filière » du Syal potentiellement porteuse d'une spécialisation, et, d'autre part, la logique "closedloop" de l'EI fortement dépendante de la diversité des activités sur le territoire. L'approche par les « paniers de biens » pourrait apporter ici des éléments de réponses;

61 - Dans le prolongement, adapter la typologie des Syal au contexte des pays en développement, notamment latino-américains, selon le type de coordination entre acteurs et les formes de stratégies collectives à l'œuvre, ouvrirait la possibilité de déterminer des «types» de démarche Syal en fonction de leur capacité à supporter la mise en œuvre d'une démarche d'EI.

\section{BIBLIOGRAPHIE}

Allenby в., 1999a, "Earth systems engineering: The role of industrial ecology in an engineered world", Journal of Industrial Ecology, vol. 2, n 3, p. 73-93.

Allenby B., 1999b, "Culture and Industrial Ecology", Journal of Industrial Ecology, vol. 3, nº 1, p. 2-4.

Allenby B., Cooper W, 1994, "Understanding industrial ecology from a biological systems perspective", Total Quality Environmental Management, vol. 3, n³, p. 343-354. 
Altieri M. A, 2002, "Agroecology: the science of natural resource management for poor farmers in marginal environments", Agriculture, Ecosystems and Environment, vol. 93, n 1-3, p. 1-24.

Altieri M. A., Nicholls C., Funes F., 2012, "The scaling up of agroecology: spreading the hope for food sovereignty and resiliency", SOCLA's Rio+20 position paper, may, $20 \mathrm{p}$.

Altieri M. A., Nicholls C.I., 2005, "Agroecology and the search for a truly sustainable agriculture", PNUE, Basic Textbooks for Environmental Training, 290 p.

Altieri M. A., Nicholls C.I., 2008, "Scaling up Agroecological approaches for Food Sovereignty in Latin America", Development, vol. 51, n 4, p. 472-480.

Altieri M.A., 2009, "Agroecology, small farms and food sovereignty", Monthly Review, vol. 61, n³, July-August, p. 102-113.

Ashton W. S., 2008, "Understanding the Organization of Industrial Ecosystems. A Social Network Approach", Journal of Industrial Ecology, vol. 12, n 1, p. 34-51.

Ashton W. S., 2009, "The Structure, Function, and Evolution of a Regional Industrial Ecosystem", Journal of Industrial Ecology, vol. 13, n 2, p. 228-246.

Astley W. G., Fombrun C. J., 1983, "Collective strategy: social ecology of organizations environments", Academy of Management Review, vol. 8, n 4, p. 576-587.

Banque Mondiale, 2008, L'agriculture au service du développement, Rapport sur le développement dans le monde, New York, $424 \mathrm{p}$.

Beaurain Ch., Brullot S., 2011, « L'écologie industrielle comme processus de développement territorial : une lecture par la proximité. », Revue d'Économie Régionale \& Urbaine, Juin, p. 313-340.

Bey Ch., 2001, "Quo Vadis Industrial ecology? Realigning the discipline with its roots", Greener Management International, $\mathrm{n}^{\circ} 34$, p. 5-42.

Bogdanski A., Dubois O., Jamieson C., Krell R., 2011, "Making integrated food-energy systems work for people and climate", FAO, Environment and natural resources management Working Paper, $\mathrm{n}$ ${ }^{\circ} 45,121 \mathrm{p}$.

Boons F., Roome N., 2000, "Industrial Ecology as a Cultural Phenomenon: On Objectivity as a Normative Position", Journal of Industrial Ecology, vol. 4, n² 2, p. 49-54.

Bouba-Olga O. et Grossetti M., 2008, « Socio-économie de proximité », Revue d'Économie Régionale \& Urbaine, $\mathrm{n}^{\circ}$ 3, octobre, p. 311-328.

Boucher F., 2012, « De la AIR a los SIAL : reflexiones, retos y desafíos en América Latina », Agroalimentaria, vol. 18, $\mathrm{n}^{\circ}$ 34, p. 79-90.

Boucher F., Carimentrand A., Requier-Desjardins D., 2003, « Agro-industrie rurale et lutte contre la pauvreté : les Systèmes Agroalimentaires Localisés contribuent-ils au renforcement des "capabilités" ? ", 3 ${ }^{\text {ème }}$ Colloque sur l'Approche des Capacités, Université de Pavie, 7-9 septembre, 20 p. Boucher F., Requier-Desjardin D., Brun V., 2010, « Syal : un nouvel outil pour le développement de territoires marginaux. Les leçons de l'alliance des agro-industries rurales de la Selva Lacandona, Chiapas. », Colloque Innovation and Sustainable Development in Agriculture and food (ISDA), Montpelier, 28-30 juin, $12 \mathrm{p}$.

Buclet N., 2011, Écologie industrielle et territoriale. Stratégies locales pour un développement durable, Presses Universitaires du Septentrion, Villeneuve d'Ascq, 309 p.

Cirad-Sar, 1996, « Systèmes agroalimentaires localisés : organisations, innovations et développement local », Rapport ATP, $n^{\circ} 134$, novembre, 27 p. 
Cohen-Rosenthal E., 2000, "A walk on the human side of industrial ecology", American Behavioral Scientist, vol. 44, n² 2, p. 245-264.

COLEIT, 2012, Colloque interdisciplinaire sur l'écologie industrielle et territoriale, Université de technologie de Troyes, 17 et 18 octobre 2012, 30 communications.

Conway G. R., 1986, Agroecosystems analysis for research and development, Winrock International Institute for Agricultural Development, Bangkok, $111 \mathrm{p}$.

Courlet C., 2008, Économie territoriale, Presses Universitaires de Grenoble, 136 p.

Daly H.E., 1991, "Elements of Environmental Macroeconomics", in R. Costanza (dir.), Ecological Economics: The Science and Management of Sustainability, Columbia University Press, New York, p. 32-46.

Deutz P., Gibbs D., 2008, "Industrial Ecology and regional development: Eco-industrial development as cluster policy", Regional Studies: The Journal of the Regional Studies Association, vol. $42, \mathrm{n}^{\circ} 10$, p. $1313-1328$.

Ehrenfeld J. R., 2000, "Industrial ecology: Paradigm shift or normal science", American Behavioral Scientist, vol. $44, \mathrm{n}^{\circ} 2$, October, p. 229-244.

Ehrenfeld J. R., 2004, "Industrial ecology: a new field or only a metaphor?", Journal of Cleaner Production, $\mathrm{n}^{\circ} 12$, p. 825-831.

Ehrenfeld J. R., 2007, "Would Industrial Ecology Exist without Sustainability in the Background?", Journal of Industrial Ecology, vol. 11, n 1, p. 73-84.

Ehrenfeld J.R., 1997, "Industrial Ecology: a new field or only a metaphor?", Journal of Cleaner Production, vol. 12, p. 825-831.

Erkman S., 1998, Vers une écologie industrielle, comment mettre en pratique le développement durable dans une société hyper industrielle ?, Institut Charles Léopold Mayer, Lausanne, 252 p.

Figuière C., Metereau R., 2012, «Écodéveloppement et souveraineté alimentaire : quels enjeux pour le Sud? », Colloque international francophone « Les représentations Nord-Sud du développement durable », IUFM d'Auvergne-Clermont Ferrand, Clermont Ferrand, 19-20 décembre, 21 p.

Fourcade C., 2006a, « Les systèmes agroalimentaires comme modalités collectives », Revue française de gestion, $\mathrm{n}^{\circ} 167$, p. 183-201.

Fourcade C., 2006b, « Les Systèmes Agro-alimentaires Localisés : des stratégies de développement local originales ? ", $23^{\text {ème }}$ Colloque annuel du Conseil canadien des PME et de l'entrepreneuriat, TroisRivières, $16 \mathrm{p}$.

Fourcade C., 2008, « Des dynamiques territorialisées novatrices : le cas des PME agroalimentaires ", Revue d'Économie Régionale \& Urbaine, n², juin, p. 193-210.

Fourcade C., Muchnik J., Treillon R., 2010, Coopération, territoires et entreprises agroalimentaires, Édition Quæ, Collection Update Sciences \& Technologies, Montpellier, 135 p.

Fournier S., Muchnik J., 2010, « L'approche "Systèmes Agroalimentaires Localisés" (SYAL), un outil d'intervention pour le développement territorial ? ", Colloque Innovation and Sustainable Development in Agriculture and Food, Montpellier, 28 juin- $1^{\mathrm{er}}$ juillet, $15 \mathrm{p}$.

Frosch R. A., Gallopoulos N. E., 1989, "Strategies for Manufacturing", Scientific American, vol. 261, Special Issue Managing Planet Earth, p. 144-152.

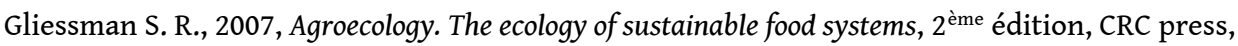
New-York, 384 p. 
Godard O., 2005, « Du développement régional au développement durable : tensions et articulations ", Chaire développement durable, Cahier n²005-16, 13 p.

Gomiero T., Giampietro M., Mayumi K., 2006, "Facing complexity on agro-ecosystems: a new approach to farming system analysis", International Journal of Agricultural Resources, Governance and Ecology, vol. 5, n² 2-3, p. 116-144.

Graedel T. E., 1994, "Industrial Ecology: definition and implementation", in Socolow R., Andrews C., Berkhout F., Thomas V. (dir.), Industrial ecology and global change, Cambridge University Press, p. 23-42.

Graedel T. E., Allenby B., 1995, Industrial Ecology, Prentice Hall, 363 p.

Hess G., 2009, « L'écosystème industriel. Difficulté épistémologique d'une telle analogie », Natures Sciences Sociétés, $\mathrm{n}^{\circ} 17$, p. 40-48.

Hill S. B., 2006, "Redesign as deep industrial ecology: lessons from ecological agriculture and social ecology", in Côté R., Tansey J., Dale A. (dir.) Linking Industry \& Ecology: A question of design, UBC Press, Vancouver, p. 29-49.

Hoffman A. J., 2003, "Linking social systems analysis to the industrial ecology framework", Organization \& Environment, vol. 16, $\mathrm{n}^{\circ}$ 1, mars, p. 66-86.

Holt Giménez E., 2008, Campesino a Campesino. Voces de Latinoamérica. Movimientos de campesino a campesino para una agricultura sustentable, SIMAS, Managua, 294 p.

Jelinski L. W., Graedel T.E., Laudise R.A., Mccall D.W., Patel C.K.N, 1992, "Industrial ecology: Concepts and approaches", Proceedings of the National Academy of Sciences of the United States of America (PNAS-USA), Colloquium Paper, vol. 89, February, p. 793-797.

Laganier R., Villalba B., Zuindeau B. 2002, « Le développement durable face au territoire : éléments pour une recherche pluridisciplinaire », Revue développement durable et territoires, dossier 1, septembre, http://developpementdurable.revues.org/774 ; DOI : 10.4000/ developpementdurable.774

Larroa R. M., 2010, «El SIAL y sus diferencias con el enfoque del desarrollo territorial en América Latina », $16^{\text {th }}$ EAAE Seminar: Spatial dynamics in agri-food systems: implications for sustainability and consumer welfare, Parme, 27-30 octobre, $9 \mathrm{p}$.

Lévesque B. 2007, « Économie plurielle et développement territorial dans la perspective du développement durable : quelques éléments théoriques de sociologie économique et de socioéconomie ", CRISES, collection Études théoriques, 75 p.

Lifset R., Graedel T. E., 2002, "Industrial Ecology: Goals and Definitions", in Ayres R. U., Ayres. L. (dir.), Handbook for Industrial Ecology, Edward Elgar, p. 3-15

Lombardi D. R., Laybourn P., 2012, Redefining Industrial Symbiosis. Crossing AcademicPractitioner Boundaries", Journal of Industrial Ecology, vol. 16, n 1, February, p. 28-37.

Maillefert M., Schalchli P., 2012, «Écologie industrielle et développement territorial : des dynamiques multiformes », Colloque de l'AFEP, 5-7 juillet, Session Régulations sectorielles et territoriales et développement durable, $16 \mathrm{p}$.

Mathews J. A., Tan H., 2011, "Progress Toward a Circular Economy in China. The Drivers (and Inhibitors) of Eco-industrial Initiative", Journal of Industrial Ecology, vol. 15, n 3, p. 435-457.

Michel J., Weiske A., Möller K., 2010, "The effect of biogas digestion on the environmental impact and energy balances in organic cropping systems using the life-cycle assessment methodology", Renewable Agriculture and Food Systems, vol. 25, n 3, p. 204-218. 
Moine A., 2006, « Le territoire comme un système complexe : un concept opératoire pour l'aménagement et la géographie. ", L'Espace géographique, n 2, tome 35, p. 115-132.

Mollard A., 2003, « Multifonctionnalité de l'agriculture et territoires : des concepts aux politiques publiques », Cahiers d'économie et sociologie rurales, $n^{\circ} 66$, p. 28-54

Mollard A., Pecqueur B., 2007, « De l'hypothèse au modèle du panier de biens et de services. Histoire succincte d'une recherche ", Économie rurale, n 300, juillet-août, p. 110-114.

Muchnik J., 2002, « Les systèmes agroalimentaires localisés : intérêt, approche, interrogations ", Colloque Syal 2002, Texte introductif, Montpellier, 16-18 octobre, 12 p.

Ometto A. R., Ramos P., Lombardi G., 2004, « Geripa, a new concept for renewable energy and food production with environmental and social concerns ", in Ortega E., Ulgiati S. (eds): Proceedings of IV biennial international Workshop Advances in Energy Studies, Unicamp, Campinas, Brazil, 16-19 June, p. 323-238.

Opoku H. N., Keitsch M. M., 2006, « Une approche objective de la durabilité ? Théories des implications scientifiques et politiques de l'écologie industrielle ", Écologie et politique, $\mathrm{n}^{\circ} 32$, p. 141-152.

Pecqueur B., 2001, «Qualité et développement territorial : l'hypothèse du panier de biens et de services territorialisés ", Économie rurale, $n^{\circ} 261$, p. 37-49.

Pretty J., Morrison J. L., Hine R. E., 2003, "Reducing food poverty by increasing agricultural sustainability in the development countries", Agriculture, Ecosystems and Environment, $n^{\circ} 95$, p. 217-234.

Requier-Desjardins D., 2007, « Systèmes agroalimentaires localisés et qualification : une relation complexe ", Colóquio Internacional sobre Desenvolvimento Territorial Sustentável (DTS), Florianopolis, Brésil, août, $12 \mathrm{p}$.

Requier-Desjardins D., 2010, «L'évolution du débat sur les SYAL : le regard d'un économiste ", Revue d'Économie Régionale \& Urbaine, $\mathrm{n}^{\circ} 4$, p. 651-668.

Rodriguez-Borray G., Requier-Desjardins D., 2005, « La multifuncionalidad de los SIALES en zonas rurales de países en desarrollo. El caso de la agroindustria panelera Colombiana », Revista Perspectivas Rurales, $\mathrm{n}^{\circ}$ 17-18, p. 113-125.

Sachs I., 1980, Stratégies de l'écodéveloppement, Éditions Économie et Humanisme, Paris, 140 p.

Sachs I., 1990, « Desarrollo sustentable, Bio-industrialización descentralizada y nuevas configuraciones rural-urbanas. Los casos de India y Brasil », Pensamiento Iberoamericano, $\mathrm{n}^{\circ} 16$, p. 235-256.

Sachs I., Silk D., 1990, Food and Energy: Strategies for sustainable development, United Nation University Press, Tokyo, 83 p.

Sarandón S., 2002, Agroecología. El camino hacía una agricultura sustentable, Ediciones Científicas Americanas, Argentina, 557 p.

Spash C. L., 2011, "Social Ecological Economics: Understanding the Past to See the Future", American Journal of Economics and Sociology, vol. 70, $n^{\circ} 2$, p. 340-375.

Theys J., 2002, « L'approche territoriale du "développement durable", condition d'une prise en compte de sa dimension sociale », Revue Développement Durable et Territoires, Dossier $1:$ Approches territoriales du Développement Durable, 18 p. 
Unesco, 2009, «Proposition d'un nouveau thème dans le contexte du Programme MAB : Les agroécosystèmes ", Conseil de Coordination International pour le programme sur l'Homme et la Biosphère (MAB), $21^{\text {ème }}$ session, Jeju, 25-29 mai, 7 p.

Vindel B., Jacquet P., 2011, « Agriculture, Développement et sécurité alimentaire », in Jacquet P., Lorenzi J-H. (dir.) Les nouveaux équilibres agroalimentaires mondiaux, Presse Universitaires de France, Collection : Les Cahiers, Le cercle des économistes, p. 73-92.

Vivien F. D. et Zuindeau B., 2001, « Le développement durable et son espace : antécédents intellectuels ", Cahiers Lillois d'économie et de sociologie, n³7, p. 11-40.

Wells P., Zapata C., 2012, "Renewable Eco-industrial Development. A Nex Frontier for Industrial Ecology?", Journal of Industrial Ecology, vol. 16, nº 5, p. 665-668.

Yami S., 2006, « Fondements et perspectives des stratégies collectives », Revue Française de Gestion, $\mathrm{n}^{\circ} 167$, p. 91-104.

\section{NOTES}

1. Une première version de ce texte a fait l'objet d'une communication aux XXVIII ${ }^{\text {èmes }}$ journées de l'ATM, du 11 au 13 juin 2012 à Orléans.

2. Paraphrasant ainsi le titre de la première section de l'article de Theys (2002) : «le territoire brique de base du développement durable ».

3. Traduction des auteurs.

4. Renaud Metereau a passé les six derniers mois de 2012 au Nicaragua pour réaliser le terrain de sa thèse. Les matériaux sont actuellement en cours d'exploitation.

5. Le projet fut à l'origine l'objet d'un programme de l'Université des Nations Unies intitulé "Food Energy Nexus Program" qui s'est déroulé entre 1983 et 1988. Les Nations-Unies demandent ensuite à Sachs et Silk d'en faire la synthèse qu'ils vont publier en 1990 "Food and Energy Strategies for Sustainable Development"(83 p.).

6. Le terme d'écologie industrielle a été traduit littéralement du terme "industrial ecology" pour lequel industrial" est entendu au sens le plus large. On ne s'intéresse pas uniquement au secteur de l'industrie mais aux sociétés industrialisées, «modernes », dans leur ensemble. Cela permet de rappeler la trans-sectorialité des démarches d'EI, inclusives, entre autres, des activités agricoles.

7. «Le déterminisme technologique se fonde sur au moins trois présuppositions. Tout d'abord, l'hypothèse d'une profonde séparation entre ce qui est technique et ce qui est social, entre la machine et l'organisation. Ensuite, la technologie s'est développée indépendamment de la société, de manière autonome et en fonction de ses propres prémisses. [...] Enfin, la technologie est un type de variable indépendante de l'évolution de la société, et basée sur de simples relations de cause à effet. » (Opoku et Keitsch, 2006 : 143-144).

8. Sachs n'a pas explicitement écrit sur l'EI, mais avant que la notion ne se popularise, il en avait déjà énoncé les principes: "les impacts négatifs des activités humaines sur l'environnement [sont] réduits moyennant le recours aux procédés et formes d'organisation de production permettant de profiter de toutes les complémentarités et d'utiliser les déchets à des fins productives » [...] «les cycles écologiques fonctionnant ici comme un paradigme de la planification. » (Sachs, 1980 : 34-35).

9. En novembre 2012, le Coleit, Colloque interdisciplinaire sur l'écologie industrielle et territoriale, est organisé par l'Université de technologie de Troyes. Trente communications dédiées à cette thématique y sont présentées. Si toutes ne s'inscrivent pas une approche socio-économique, elles postulent néanmoins toutes sur le nécessaire ancrage territorial des démarches d'EI. 


\section{RÉSUMÉS}

Afin de poser les jalons de la réflexion sur un «développement rural local durable », le Syal (Système Agroalimentaire Localisé) est ici considéré comme un point de départ pertinent. Afin de rendre plus «durable » le projet de développement local dont il est porteur, il va être enrichi dans un premier temps par des outils dédiés aux activités agricoles et à visée spécifiquement environnementale, l'agroécologie et l'IFES (Integrated Food Energy System). L'approche socioéconomique de l'écologie industrielle vient ensuite compléter la proposition en systématisant notamment le principe de bouclage des flux.

Laying the groundwork for "sustainable local rural development", the Local Agri-Food System is here considered as a relevant starting point. To make this local development strategy more "sustainable", firstly, it will be completed by specific environmental tools from the agricultural sector, agroecology and the IFES (Integrated Food Energy System). The socio-economic approach of industrial ecology is then used to wrap up this proposal by systematically including the principle of closed-loop flows.

\section{INDEX}

Keywords : rural development, sustainability, local approach, industrial ecology, local agri-food system, agroecology, IFES

Mots-clés : développement rural, durabilité, territoire, écologie industrielle, Syal, agroécologie, IFES

\section{AUTEURS}

\section{RENAUD METEREAU}

Renaud Meterau est doctorant allocataire au sein du Centre de Recherche en Économie de Grenoble (CREG). Sa thèse porte sur l'intégration de la dimension environnementale dans les stratégies de développement rural local. Université de Grenoble, renaud.metereau@upmfgrenoble.fr

\section{CATHERINE FIGUIÈRE}

Catherine Figuière est Maître de Conférences, habilitée à diriger les recherches en économie. Elle dirige actuellement le Centre de Recherche en Économie de Grenoble de l'Université Pierre Mendès France. Ses travaux et ses publications récentes portent pour une grande part sur le développement durable. Elle est titulaire de plusieurs cours sur l'économie politique du développement durable dans sa Faculté. Université. Grenoble Alpes, catherine.figuiere@upmfgrenoble.fr 